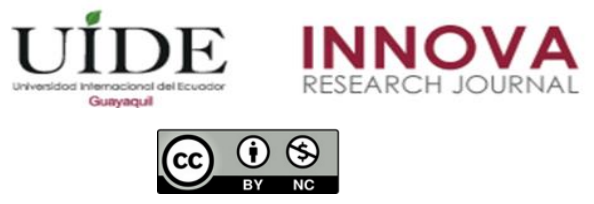

INNOVA Research Journal, ISSN 2477-9024

(Septiembre-Diciembre 2020). Vol. 5, No.3 pp. 233-244

DOI: https://doi.org/10.33890/innova.v5.n3.2020.1386

URL: http://revistas.uide.edu.ec/index.php/innova/index

Correo: innova@uide.edu.ec

\title{
Certificación Turística como Herramienta de Diferenciación Clave para las Playas: Caso Playa Chipipe. Salinas- Ecuador
}

\section{Tourist Certification as a Key Differentiation Tool for the Beaches: Playa Chipipe Case. Salinas- Ecuador}

Franklin Marcelo Mora Méndez

https://orcid.org/0000-0002-1618-898X

Universidad Internacional del Ecuador, Ecuador

Segundo Ronal García Castro

Universidad de Guayaquil, Ecuador

Edison Fernando Chiriboga Cisneros

https://orcid.org/0000-0002-5764-8065

Universidad de Guayaquil, Ecuador

Autores para correspondencia: mora53franklin@hotmail.com; ron182garcia@gmail.com; edison.chiribogac@ug.edu.ec

Fecha de recepción: 31 de marzo del 2020 - Fecha de aceptación: 16 de agosto del 2020

\section{Resumen}

El turismo de sol y playa sigue siendo de gran trascendencia mundial como motivador de viajes turísticos. La zona costera del pacifico ecuatoriano, posee una diversidad de playas con atractivos naturales y culturales que favorecen la motivación de los turistas. El incremento de visitantes, sumado a la saturación de planta turística y demás componentes del sistema turístico pueden ocasionar serios impactos negativos, por lo cual implica una gestión orientada con criterios de sostenibilidad que garantice mantener los estándares de calidad de los servicios y sus recursos. La investigación consistió en evaluar la playa Chipipe del cantón salinas apoyada en los parámetros de la norma ecuatoriana INEN 2631, para su certificación como primera playa turística certificada en el Ecuador. La metodología utilizada se fundamentó básicamente en el trabajo de campo a través de técnicas de observación y recopilación de información directa de los actores locales involucrados en el sector. Se obtuvo como resultado que la playa de Chipipe aplica para una certificación de clase "C" según la norma, cumpliendo con los indicadores de autogestión, seguridad, zonificación e infraestructura.

Palabras Claves: certificación turística; Ecuador; turismo de sol y playa; turismo sostenible; calidad turística

\footnotetext{
Abstract

Sun and beach tourism continue to be of great worldwide importance as a motivator of tourist trips. The Ecuadorian Pacific coastal zone has a diversity of beaches with natural and cultural resources 
that motivates tourists. The increase in visitors, added to the saturation of tourist services and other components of the tourism system, can cause serious negative impacts, which means that coastal zone management should be oriented with sustainability criteria that guarantee the quality standards of the services and available resources. This research consisted of evaluating Chipipe beach in the city of Salinas in order to look for its certification supported by the parameters of the Ecuadorian standard INEN 2631. The methodology used was based on field work through observation techniques and direct information gathering from involved local actors. As a result, Chipipe beach applied for a class " $C$ " certification according to the standard, complying with several indicators such as self-management, security, zoning and infrastructure.

Key words: tourism certification; Ecuador; sun and beach tourism; sustainable tourism, tourist quality

\section{Introducción}

El sector turístico tiene un rápido crecimiento en los países desarrollados y en vías de desarrollo a tal punto que se ha convertido en un fenómeno socioeconómico importante en el siglo XX y XXI. La Organización Mundial del Turismo (OMT) señala que la llegada de turistas internacionales en el año 2018 creció a un 6\% dando como resultado 1.4 mil millones turistas y según el índice de confianza de la OMT se prevé un crecimiento del 3\% al 4\% de llegadas de turistas internacionales en el año 2019 en todo el mundo (Tourism \& Unwto, 2019).

Ecuador tiene un crecimiento turístico importante en los últimos años, en el año 2018 llegaron 2427.660 según el ministerio del ramo. EEUU como turismo emisor tuvo un crecimiento del 43. 8\% en el año 2018 según cifras del Ministerio de Turismo del Ecuador (MINTUR, 2018).

Efectivamente la actividad produce beneficios en el desarrollo local producto de flujos turísticos a través de visitantes nacionales y extranjeros. Estos aspectos positivos de la actividad han permitido dinamizar las economías locales, comprender la funcionalidad de los territorios vistos como espacios rurales alternativos para generar el desarrollo sostenible. En este sentido, se han creado herramientas de turismo sostenible como las certificaciones de playas, que permiten medir los estándares de calidad en el servicio, manejo, seguridad, ambiente, salud etc., en estos destinos.

Las certificaciones de playas en Latinoamérica son relativamente nuevas. En el año 1996 Costa Rica implementa el programa Bandera Azul Ecológica (BAE), adoptado en base a la filosofía de la certificación de playas Bandera Azul (BA) emitida en Francia en 1987. El Ministerio de Turismo en el año 2013, lanza la iniciativa del programa de certificación de playas limpias a fin de estandarizar la calidad turística de las playas en Ecuador. Ante esto, existe la necesidad de implementar mejoras en la calidad que promuevan el aprovechamiento turístico de las playas y que el compromiso lo asuman los organismos gubernamentales, comunidad y visitantes.

La playa Chipipe es muy visitada por los turistas nacionales y extranjeros, los gestores turísticos han logrado aprovechar turísticamente el destino desde los años noventa, cuando se 
dan las primeras corrientes de turistas nacionales al lugar atraídos por los recursos naturales, escena paisajística, aguas medicinales de los baños de San Vicente (S. GAD, 2019).

Actualmente el balneario de salinas es un centro turístico de primer orden. No obstante, existen problemas de planificación turística, impactos ambientales negativos que resultan focos infecciosos, degradación de los ecosistemas producto del mal manejo de aguas residuales, desechos etc. Así también las actividades antrópicas en los sectores como la minería, pesca y turismo (S. E. GAD, 2019).

El presente trabajo consiste en la realización de una evaluación turística aplicada en la playa Chipipe del Cantón Salinas, ubicada en la provincia de Santa Elena. Se inició identificando cada uno de los estándares que están relacionados con el enfoque del turismo sostenible como el ámbito social, económico y ambiental de la zona de estudio, basado en la norma INEN ecuatoriana 2631 para la certificación de las playas (INEN., 2012)

El objetivo de la investigación es evaluar la Playa Chipipe del Cantón Salinas para conocer si cumple con los indicadores de la norma INEN y que posteriormente logre ser playa turística certificada de la provincia de Santa Elena. Para lo cual, se tuvo que actualizar la línea base turística del área de estudio a fin de identificar las potencialidades y debilidades que posee la playa. Se analizó la metodología de aplicación para la certificación de playas turísticas sostenibles reconocidas en el ámbito internacional y de Latinoamérica. Al final se aplicó los parámetros de certificación de la norma INEN ecuatoriana 2631, para la playa Chipipe, con el objetivo de evaluar la situación de la sostenibilidad turística y así alcanzar una certificación.

En un estudio realizado en la playa Punta Carnero sobre un análisis de la capacidad de carga turística y factores que inciden en la percepción de los visitantes, asi también apectos del entorno económico, social, ambiental (Hurtado \& Rodriguez, 2007). información relevante que ayuda a contrastar la data de destinos costeros para la investigación de la playa Chipipe.

La certificación de la playa es el punto de partida para promover y ejecutar estrategias que ayuden atraer inversión en el ámbito de turismo sostenible, ya sea esta pública o privada y que contribuya al fortalecimiento de la actividad de un turismo sostenible en la playa. El beneficio de playa certificada motivará a los turistas ya sean extranjeros o nacionales a experimentar de un turismo más respetuoso con el ambiente y a mejorar la calidad de vida de la comunidad local, generar experiencias satisfactorias en visitantes y conservar los recursos naturales y culturales.

\section{Marco Teórico}

La adopción de sistema de gestión de la calidad y certificación de las playas supone un punto de inflexión en la actualidad a fin mantener los servicios ecosistémicos, las playas contemplan un sistema multidimensional las cuales están conformadas por subsistemas como socio culturales, ambientales y de gestión (Yepes, 2007). El escenario actual de la certificación de playas a nivel internacional está debidamente normalizado, implementado como en el caso de España, Francia, EEUU, México etc., como ejemplo en el caso de España desde 1969, ya 
contaba con un plan de ordenación general de Playas de la ley de Costas, empleado en la comunidad de Valencia de forma masiva para los destinos costeros.

Como antecedentes a la investigación se recopilaron datos que faciliten la comprensión metodológica y teórica de la certificación de playas, guias básicas que ayudan a entender las generalidades de las certificaciones de playas y poder conocer los requisitos principales e importantes que encierra una certificación, además de los pasos a seguir. De esta manera enseña mediante una variedad de ejercicios, estimar el nivel que tiene una playa para la certificación, demostrando la organización, responsabilidad y esfuerzo que se requiere para lograr una certificación (Botero, Zielinski, \& Noguera, 2012)

También se analizó estudios que incorporan de la percepción de los usuarios en la certificación de playas limpias, a fin de obtener un enfoque integral que logre garantizar seguridad y salud de los visitantes y tomar en cuenta las particularidades que poseen, las mismas que son motivadores para su uso, además que surgieren incorporar la percepción de los usuarios para atender las respuestas a los problemas que los certificadores de playas dejan pasar por alto. Se aplicaron dos métodos de certificación en tres playas de México (Condensa, Revolcadero y Caleta) dando como resultado que las playas antes mencionadas no son certificables, pero que según los usuarios las tres playas presentan características diferentes. Se elaboró una propuesta sobre un plan de manejo para cumplir con el insuficiente cumplimiento de indicadores de las playas, esperando que con la incorporación de la percepción de los usuarios, ayuden al proceso de certificación de las tres playas (UNESCO, 2012)

Botero, S., en colaboración con los autores Zielinski, S. y Noguera, L. En el año 2012 publicaron un estudio titulado Esquema de Certificación de Playas en América Latina: diagnóstico de una herramienta de manejo integrado costero, presentan una comparación de ocho esquemas de certificación de playas en América latina, en el marco de evaluación y de los útiles que son estas herramientas dentro del manejo integral costero. Además, sostienen como conclusión que las variables de prevención, seguridad turística y calidad ambiental optima permiten sostener los principios del desarrollo sostenible de sol y playa.

El análisis de los principios del desarrollo sostenible demuestra que en el turismo de sol y playa el interés se basa en la seguridad y la óptima calidad ambiental, es decir un comportamiento preventivo y cauto (Botero et al., 2012)

Es fundamental el aporte de evaluación de ocho certificaciones en Iberoamérica amparado en un estudio denominado la utilidad de los esquemas de certificación de playas para el manejo integrado costero. Se construyeron cuatro indicadores, participación en la gestión, gestión eficiente de playas, promoción de la participación pública y la medición de capacidad de carga, dando como resultado tres indicadores con nivel bajo y uno con nivel medio (Botero, 2009).

En el panorama ecuatoriano y específicamente en la zona de estudio es evidente el déficit de cumplimiento de indicadores para la certificación de las playas, en este sentido se recopiló información tanto en el cantón salinas como en la zona de influencia ,como por ejemplo el estudio plan de desarrollo de actividades acuáticas de la playa San Lorenzo, cantón Salinas, 
provincia de Santa Elena, que propone fortalecer el sector turístico de San Lorenzo ya que ayudaría a mejorar la calidad de vida de los habitantes, incrementando su economía a través de una adecuada coordinación de las actividades turísticas especialmente los deportes acuáticos, involucrando a la comunidad, el sector público y privado.

Además, el trabajo realizado por el Ministerio de Turismo y la Consultora Ecosambito titulado implementación de las normas técnicas de certificación de playa turística que fue realizada en el 2009, en el cual se ejecutó los primeros avances y pasos para la implementación de la Norma Técnica de Playa Turística, además de desarrollar las herramientas que ayudan a la evaluación de la playa de Chipipe.

La investigación denominada plan estratégico de promoción turística de la playa de Muisne provincia de Esmeraldas para incentivar el desarrollo turístico, se analizaron diferentes factores que le permitió conocer sus fortalezas y debilidades para la mejora del destino, entre estos por ejemplo un aspecto importante es la cultura afro ecuatoriana principal atractivo, con todo lo que engloba su folclore y gastronomia, indicadores sociales que sirve de motivadores a los visitantes, así también las fortalezas que posee la comunidad para apostar por el turismo (Salguero, 2012). El análisis de factores determinantes para el cumplimiento de la certificación de una playa sirvió como elemento a tomar en cuenta en Chipipe.

Otro proyecto que ayudó a la investigación es el conocimiento de varios aspectos sociales, económicos y de gestión fundamentalmente que se formula en el plan integral de desarrollo y promoción turística del cantón San Vicente, publicado en el año 2012. Uno de los indicadores relevantes para este tipo de destinos costeros es la gobernaza turística, paralelo a ello existe deficiente capacitación en la comunidad principalmente en los prestadores de servicios turísticos, El estudio tiene como objetivo promocionar a escala internacional ya que al momento el $90 \%$ de turistas son extrajeros que utilizan de paso para dirigirse a playas más consolidadas como lo es la playa de Canoa (Manrique, 2012).

En la Isla Puna se elaboró un plan estratégico que apunta a difundir turísticamente cuatro comunas de la Isla ubicadas en el golfo de Guayaquil, fue pulicado en el año 2014 , en la investigación se analizaron varios enfoques, como la política turística, superestructura, la accesibilidad a los principales atractivos de este destino y especialmente la capacitación como indicador de la calidad de los servicios que se prestan en la zona de estudio, a fin de obtener un panorama turístico se desarrolló inventarios de cada uno de los atractivos para conocer las fortalezas y debilidades que posee la Isla Puna. (Pazmiño \& Yagual, 2014).

\section{Norma Técnica Ecuatoriana NTE INEN 2631 (Ecuador)}

Es una norma técnica elaborada por el Instituto Ecuatoriano de Normalización (INEN) que pretende dar certificación a las playas que logren cumplir con todos los requisitos y que manifiesten que están en óptimas condiciones para brindar a los visitantes un servicio de calidad. (INEN, 2012)

Esta norma fue creada en el año 2007 y está basado en el modelo internacional llamado "Bandera Azul". En el Ecuador se han adaptado los requerimientos del programa para poder 
certificar las playas en tres categorías que son: Clase "C": Autogestión, manejo de información, organización local y parámetros mínimos de seguridad. Clase "B": Mejoras relacionadas con infraestructura y seguridad integral. Clase "A": tiene que ver con variables de calidad ambiental, de seguridad turística el saneamiento de destinos costeros y de servicio. Sostenibilidad de los recursos naturales.

Para poder lograr cualquiera de estas categorías, cada playa tendrá que cumplir los requisitos que presenta la Norma Técnica Ecuatoriana NTE INEN 2631:2012, Turismo. Playas. Requisitos de certificación turística. Que trata sobre la gestión de desechos en las playas, buenas prácticas ambientales en la gestión del agua, regulaciones del comercio, infraestructura óptima, conservación de la biodiversidad, seguridad turística, prestación de servicios, información y medios de comunicación. (INEN, 2012).

Se desarrolló un plan piloto en el año 2009 en las cuales se seleccionaron a 4 playas entre estas: canoa, libertador Simón Bolívar, las Palmas y la playa conocida como el Arenal, el objetivo trabajar a fin de cumplir los requisitos de certificación. En el año 2012 esta norma de certificación se dio a conocer a todas las comunidades costeras del país para que logren proponer algunas de sus playas para que certifiquen como Playa turística.

\section{Turismo en el Cantón Salinas}

El Ecuador se caracteriza por ofrecer un producto turístico de naturaleza los cuales posee relevancia cultural, turismo de sol y playa, turismo de aventura, ecoturismo y turismo de negocios. Según el estudio realizado por el Ministerio de turismo del Ecuador la tipología de turismo de sol y playa están dentro de los productos más comercializados ocupando la tercera posición después de ecoturismo y particularme Galapagos (MINTUR, 2018).

Una de las estrategias que tiene el ministerio de turismo a través del plan de desarrollo turístico PLANDETUR 2020, el turismo de sol y playa sostenible se presenta como un producto de oportunidad para el mercado internacional (MINTUR, 2007)

El Balneario de Salinas se ubica a $142 \mathrm{~km}$ del puerto principal Guayaquil, el mismo se prolonga desde la península de Santa Elena hasta la punta más sobresaliente conocida como Puntilla de Santa Elena en el occidente. Al norte, oeste y sur limita con las aguas del Océano Pacífico y al este con el cantón Santa Elena. La extensión de su playa es de aproximadamente 15 $\mathrm{km}$.

El nombre Salinas proviene de las enormes minas de sal yodada que se explotan en su suelo solitario desde épocas pre coloniales. Además, el sitio se ha caracterizado por ser el lugar de diversión preferido por los ecuatorianos y un importante centro turístico, ya que cuenta con diversidad de establecimiento de alimentos y bebidas como: clubes, bares, hoteles, restaurantes, casinos y hasta centros deportivos. Es el lugar ideal para practicar deportes acuáticos como vela, buceo, jet ski, y pesca deportiva (S. GAD, 2019).

Salinas esta entre las zonas donde se presenta alta diversidad de vida silvestre de la costa ecuatoriana, a escasos metros de la playa está la Reserva de Producción de Fauna Marino Costera 
Puntilla de Santa Elena. Las formaciones vegetales están compuestas por: matorral seco de tierras bajas, matorral seco litoral, espinar litoral (Cerón, Palacios, Valencia, \& Sierra, 1999)

En cuanto a la riqueza faunística de especies de peces el instituto Nazca en la Puntilla registró 86 especies de peces, 160 especies de aves, 16 especies de mamíferos marinos. Los registros que presenta Escalante, et al.(2008) sobre las tortugas marinas hay tres especies entre ellas tortuga carey (Eretmochelys imbricata) la cual se encuentra en peligro crítico de extinción según la Unión Internacional para la conservación de la naturaleza (UICN) (MAE, 2009).

La planta turística y facilidades en el cantón Salinas han permitido un crecimiento turístico constante, según reporte de las estimaciones del turismo en el Ecuador en el año 2019 en épocas de feriado Salinas se ubica en los tres principales destinos del país. En Semana Santa ocupó el 3,38\% de llegada de turistas de 576 mil turistas a nivel nacional, en feriado de carnaval obtiene un 5\% de visitas de 723 mil turistas a nivel nacional y en el feriado de fin de año obtiene $4 \%$ de turistas a nivel nacional superado por Quito y Guayaquil los principales destinos turísticos en el Ecuador continental, con un gasto aproximado de 8 millones de dólares en los tres feriados (MINTUR, 2019)

En los meses de febrero, marzo y abril temporada alta, Salinas cuenta con un mercado emisor fijo como lo es Guayaquil, Machala y Cuenca en temporada de vacaciones de la región Andina. En cuanto al turismo internacional Salinas recibe turistas internacionales provenientes principalmente de Europa, Brasil y Norteamérica, con visitas anualmente alrededor de 150.000 turistas internacionales (S. GAD, 2019)

\section{Metodología}

La metodología de la investigación es de carácter mixto, se usó el método cuantitativo y cualitativo. Desde la perspectiva cuantitativa se empleó varios métodos para la recolección de datos, por ejemplo, datos estadísticos de visitantes en la playa, también se aplicaron encuestas estructuradas que permitió obtener información relevante de los fenómenos y factores causales. La observación de campo fue fundamental para el estudio, ya que al estudiar los fenómenos insitu se cuantificó el impacto de las actividades turísticas y las deficiencias en el cumplimiento de los indicadores de la norma. Para la aplicación de las encuestas se usaron cuestionarios como guías de observación y lista de chequeo.

Desde la perspectiva cualitativa se realizó entrevistas a los turistas mediante las técnicas de la entrevista dirigida y no estructurada. Esta técnica permitió obtener información más detallada y real mediante un diálogo abierto para captar las percepciones del sujeto de la investigación en este caso de los actores involucrados directamente en la gestión del turismo en la zona de estudio. Además, se utilizó entrevistas de profundidad con el fin de obtener datos de las experiencias de los visitantes.

Se consultó a los turistas sobre la percepción que tienen con respecto a ciertas situaciones de la playa, valoradas del 1 al 5 según la escala de Likert. La encuesta se la realizó a 384 visitantes en un fin de semana en temporada alta. El investigador emitió su criterio sobre aspectos evaluados en esta metodología. 


\section{Resultados}

De los 57 ítems necesarios para alcanzar la certificación en la playa urbana de Chipipe, un ítem no aplica debido a la falta de información sobre muestreos de desechos por cuadrante según la norma INEN para esta playa; de los restantes 56 ítems, la playa cumple con 33 e incumple con 23 ítems respectivamente.

\section{Tabla 1}

Resumen de cumplimiento de requisitos según la norma INEN 2631

\begin{tabular}{|c|c|c|c|c|}
\hline $\begin{array}{c}\text { Ejes de } \\
\text { evaluación }\end{array}$ & $\begin{array}{l}\text { Requisitos } \\
\text { necesarios }\end{array}$ & $\begin{array}{c}\text { Requisitos que no } \\
\text { aplica }\end{array}$ & $\begin{array}{l}\text { Requisitos } \\
\text { cumplidos }\end{array}$ & $\begin{array}{l}\text { Requisitos no } \\
\text { cumplidos. }\end{array}$ \\
\hline Recursos hídricos & 5 & 0 & 4 & 1 \\
\hline Desechos & 11 & 1 & 10 & 0 \\
\hline Seguridad & 9 & 0 & 2 & 7 \\
\hline Vigilancia & 3 & 0 & 3 & 0 \\
\hline Zonificación & 3 & 0 & 3 & 0 \\
\hline $\begin{array}{c}\text { Vehículos } \\
\text { motorizados }\end{array}$ & 3 & 0 & 2 & 1 \\
\hline $\begin{array}{c}\text { Seguridad en } \\
\text { general }\end{array}$ & 2 & 0 & 1 & 1 \\
\hline $\begin{array}{c}\text { Servicios } \\
\text { higiénicos }\end{array}$ & 3 & 0 & 1 & 2 \\
\hline $\begin{array}{l}\text { Facilidades de } \\
\text { comunicación }\end{array}$ & 4 & 0 & 1 & 3 \\
\hline $\begin{array}{c}\text { Servicios } \\
\text { discapacitados }\end{array}$ & 1 & 0 & 0 & 1 \\
\hline Infraestructura & 3 & 0 & 3 & 0 \\
\hline $\begin{array}{c}\text { Conservación } \\
\text { recursos } \\
\text { naturales }\end{array}$ & 4 & 0 & 2 & 2 \\
\hline Señalética & 3 & 0 & 0 & 3 \\
\hline $\begin{array}{l}\text { Educación } \\
\text { ambiental }\end{array}$ & 3 & 0 & 1 & 2 \\
\hline Total & 57 & 1 & 33 & 23 \\
\hline
\end{tabular}

Nota. Esta tabla muestra el nivel de cumplimiento de la playa Chipipe según los ejes de evaluación de la norma INEN 2631.

Los ejes con mayor nivel de cumplimiento en esta playa son los de desechos sólidos (100\%), vigilancia (100\%), el eje de infraestructura (100\%), y el eje de zonificación (100\%), y los de menor nivel de cumplimiento son los ejes de señalética $(0 \%)$ y de servicios de discapacitados $(0 \%)$.

A continuación, se exponen los resultados de la playa evaluada con respecto al cumplimiento de la norma de certificación para todas las clases. Las figuras muestran los resultados obtenidos de la evaluación de la matriz en la que se describe, la norma a evaluar, el método de evaluación y cumplimiento utilizado. 
Figura 1.

Resumen de evaluación de la playa en los principales ejes de la certificación INEN 2631.

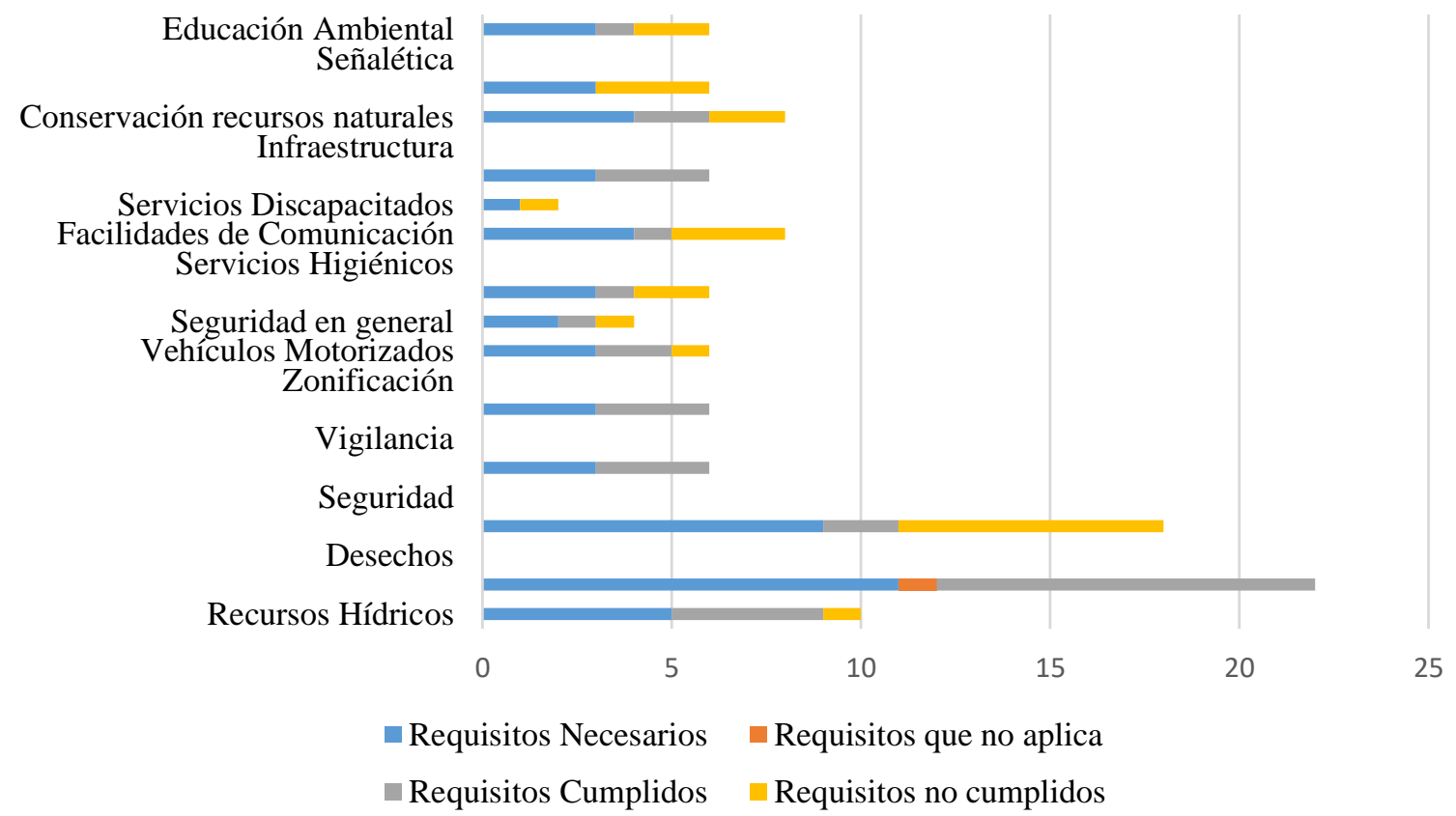

Nota. El gráfico representa el nivel de cumplimiento entre los requisitos necesario y cumplidos. Fuente elaboración propia

\section{Figura 2.}

Evaluación de la playa Chipipe en cada uno de los indicadores de la certificación INEN 2631.
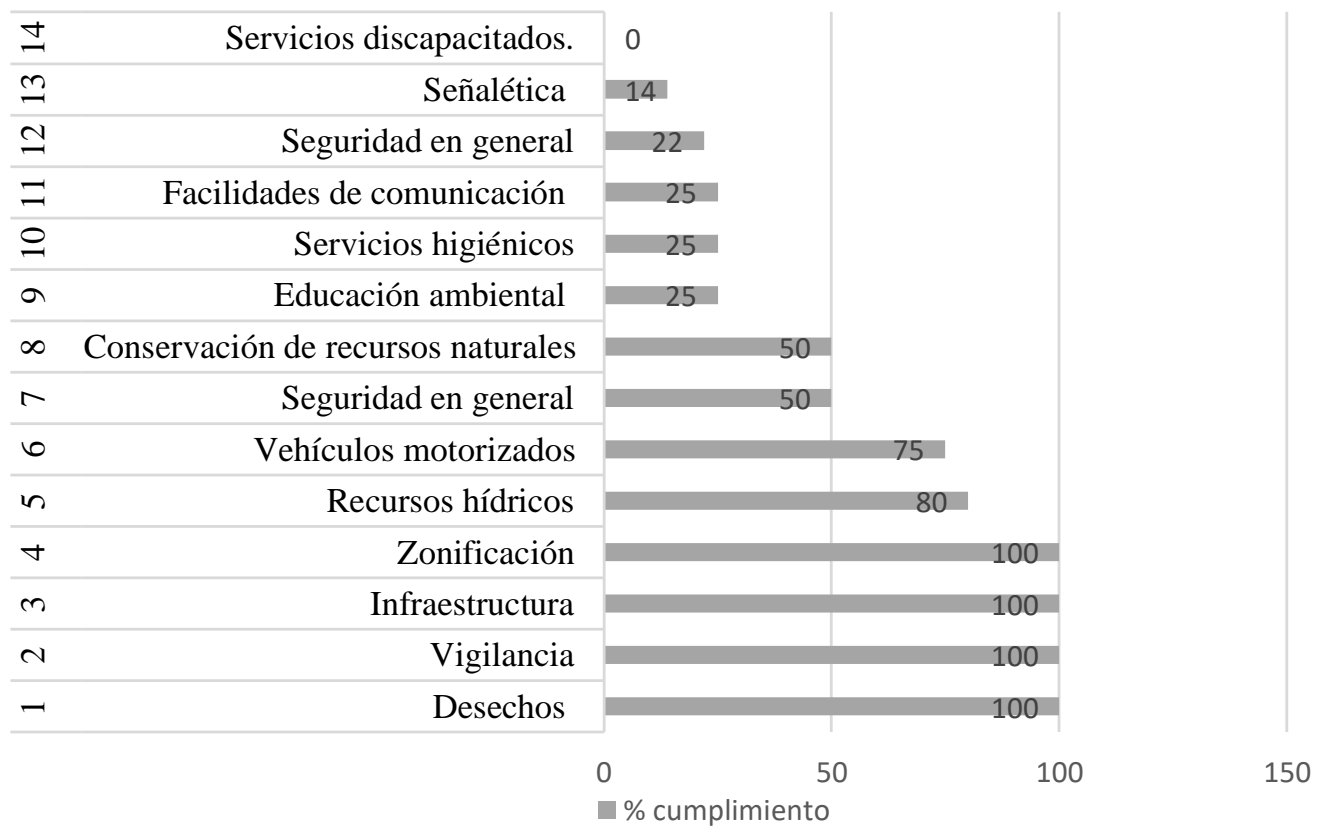

Nota. El gráfico representa el nivel de cumplimiento desde $0 \%$ al $100 \%$ de cumplimiento de los indicadores según la norma. Fuente elaboración propia. 
El porcentaje total de cumplimiento fue del $59 \%$ además con estos resultados la playa Chipipe del cantón Salinas podría obtener una certificación INEN 2631 clase C ya que cumple con los estándares de autogestión, vigilancia, zonificación e infraestructura.

\section{Conclusiones}

La evaluación de la playa Chipipe pone de manifiesto la importancia de lograr la certificación turística de las playas en el Ecuador. En este artículo se hace visible los problemas de la investigación turística que es la falta de información científica de gestión del turismo y los impactos que genera producto de la visitación en las playas.

Para la evaluación de Chipipe se realizó una línea base a fin de recopilar datos a través de estudios preliminares, encuestas, entrevistas, observación de campo, la misma que permitió una evaluación más apegada a la realidad de forma integral, sin embargo, en el área de estudio existen insuficientes estudios previos como sistema de manejo de visitantes u otra herramienta como límite de cambio aceptable, información que ayudaría a evaluar indicadores de gestión de visitantes. Así también, no hay documentos de zonificación turística que contribuya a medir indicadores de planificación y gestión de turismo.

En estudios como el Plan de desarrollo de ordenamiento territorial de cantón Salinas ya se menciona que la playa Chipipe se ubica en una zona el cual presenta un nivel de riesgo alto favorables para la ocurrencia de procesos de inundación (S. GAD, 2019), en este sentido es fundamental mejorar los niveles de seguridad que en la evaluación presentan niveles insuficientes el 78\% no cumple; de tal manera que es urgente realizar planes de gestión de crisis y contingencia.

Este artículo presenta resultados de carácter académico porque permite comprender a los planificadores turísticos la importancia de desarrollar información previa a fin de lograr el cumplimiento de indicadores que son fundamentales para mejorar la calidad de los servicios, ejemplo para los visitantes con discapacidades los resultados son negativos, la evaluación es deficiente $0 \%$ de cumplimiento.

Esta investigación tiene algunas limitaciones como por ejemplo que la encuesta se realizó a los visitantes que estaban disfrutando de la playa, evidentemente que aquellos tienen una perspectiva diferente a los visitantes que ya conocen y tuvieron algún inconveniente en cuanto a las facilidades turísticas, en este sentido puede existir un sesgo en la percepción, por lo tanto, para evaluaciones futuras, se deberá incluir las encuestas a visitantes que regresan por segunda vez o más, una encuesta estratificada. Igualmente queda pendiente realizar los muestreos del agua de la playa según indica la metodología de la certificación.

La implicación práctica que tiene esta evaluación es que ayuda a entender a los gestores turísticos la importancia de evaluar las playas y lograr obtener certificaciones que contribuyan a la planificación holística e incluyente y que ayude a la toma de decisiones para garantizar la satisfacción de los visitantes y lograr la sostenibilidad turística de los destinos costeros. 


\section{Bibliografía}

Botero, C. (2009). Utilidad de los esquemas de certificación de playas para el manejo integrado costero. Evaluación de ocho certificaciones en Iberoamérica. Santiago de Cuba, Cuba. (4), 27-41. Retrieved from http://www.redalyc.org/articulo.oa?id=181317813003

Botero, C., Zielinski, S., \& Noguera, L. (2012). Esquemas de Certificación de playas en América Latina: Diagnóstico de una herramienta de Manejo Integrado Costero. (June 2012), 29211. Retrieved from http://tiny.cc/vx5ylz

Cerón, C., Palacios, W., Valencia, R., \& Sierra, R. (1999). Formaciones Naturales de la Costa del Ecuador. En Propuesta Preliminar de un Sistema de Clasificación para el Ecuador Continental.Proyecto INEFAN/GEF-BIRF y EcoCiencia. Quito, Ecuador. Retrieved from file:///Users/mac/Downloads/Propuesta_preliminar_de_un_sistema_de_cl.pdf

GAD, S. (2019). Plan de Desarrollo y Ordenamiento Territorial del cantón Salinas. Ecuador. 232. Retrieved from http://tiny.cc/n73wlz

GAD, S. E. (2019). Plan de Ordenamiento Territorial- Canton Santa Elena 2014-2019. Ecuador. Retrieved from http://tiny.cc/wb3wlz

Hurtado, L., \& Rodriguez, M. (2007). Estudio de Capacidad de Carga de la Playa Punta Carnero del Cantón Salinas y Propuestas para mejorar su Desarrollo Turístico. Guayaquil. Ecuador. $\quad$ Retrieved from https://www.dspace.espol.edu.ec/bitstream/123456789/4659/1/7180.pdf

INEN. (2012). Turismo. Playas. Requisitos de Certificación Turística. Quito.Ecuador.

MAE. (2009). Plan de manejo Reserva de Producción Faunística Marino Costera Puntilla de Santa Elena. I, 130. Retrieved from file:///Users/mac/Downloads/Reserva de Producción Faunística Marino Costera Puntilla de Santa Elena pdf

Manrique, C. (2012). Pla Integral de Desarrollo y Promoción Turística del cantón San Vicente como medio efectivo para el mejoramiento económico y social de los pobladores de la zona. Guayaquil -Ecuador. (Universidad Católica Santiago de Guayaquil.). Retrieved from http://repositorio.ucsg.edu.ec/bitstream/3317/362/1/T-UCSG-PRE-ESP-AETH-80.pdf

MINTUR. (2007). Plan estratégico de desarrollo de turismo sostenible para Ecuador "PLANDETUR 2020”. $106 . \quad$ Retrieved from https://jorgepaguay.files.wordpress.com/2012/03/plandetur.pdf

MINTUR. (2018). Llegadas y Salidas Internacionales en Ecuador Visitas por nacionalidad y residencia.Quito Ecuador. Retrieved from https://servicios.turismo.gob.ec/index.php/turismo-cifras/2018-09-19-17-01-

51/movimientos-internacionales

MINTUR. (2019). Boletín Feriado. Estimaciones preliminares del turismo en el Ecuador. Feriado carnaval 2019. Retrieved from https://servicios.turismo.gob.ec/descargas/Turismocifras/Publicaciones/Feriados/2019/FERIADO-CARNAVAL.pdf

Pazmiño, J., \& Yagual, K. (2014). "Plan estratégico de promoción turística de cuatro comunas de la Isla Puná para potenciar los recursos naturales y promover el desarrollo turístico del sector". (Universidad Católica de Santiago de Guayaquil.). Retrieved from http://192.188.52.94/bitstream/3317/2382/1/T-UCSG-PRE-ESP-AETH-198.pdf

Salguero, D. (2012). Plan Estrátégico de Promoción Turística de la Playa de Muisne, Provincia de Esmeraldas para Incentivar el Desarrollo Turístico de este sector. (Universidad Católica Santiago de Guayaquil.). Retrieved from http://repositorio.ucsg.edu.ec/bitstream/3317/305/1/T-UCSG-PRE-ESP-AETH-43.pdf

Esta obra se comparte bajo la licencia Creative Common Atribución-No Comercial 4.0 International (CC BY-NC 4.0) Revista de la Universidad Internacional del Ecuador. URL: https://www.uide.edu.ec/ 
Tourism, W., \& Unwto, O. (2019). 2018: International tourist arrivals worldwide reach 1 . 4 billion two years ahead of forecasts. 17(1), 1-5.

UNESCO. (2012). Costas. Revista Iberoamericana de Manejo Costero Integrado. 1. Retrieved from https://unesdoc.unesco.org/ark:/48223/pf0000216917

Yepes, V. (2007). Gestión del uso y explotación de las playas. Valencia España. 241-254. Retrieved from file:///Users/mac/Downloads/document (1).pdf 